\title{
miR-96 induces cisplatin chemoresistance in non-small cell lung cancer cells by downregulating SAMD9
}

\author{
LIN WU, XINGXIANG PU, QIANZHI WANG, JUN CAO, FANG XU, LI XU and KANG LI \\ Department of Thoracic Medicine, The Affiliated Cancer Hospital of Xiangya School of Medicine, \\ Central South University, Changsha, Hunan 410003, P.R. China
}

Received January 5, 2015; Accepted September 24, 2015

DOI: $10.3892 / \mathrm{ol} .2015 .4000$

\begin{abstract}
Cisplatin is effective as a single agent or in combination with other drugs for the treatment of non-small cell lung cancer (NSCLC). A concerning clinical challenge with cisplatin-based NSCLC chemotherapy is the intrinsic and acquired chemoresistance to cisplatin. The sterile $\alpha$ motif domain-containing (SAMD9) gene has been reported as a potent tumor suppressor gene that inhibits tumorigenesis and progression of NSCLC. microRNAs (miRNA) have been revealed to play important roles in the regulation of cancer chemoresistance. To the best of our knowledge the present study explored the role of miRNA/SAMD9 signaling in regulating cisplatin chemoresistance in NSCLC for the first time. Out of the several candidate miRNAs predicted to bind the 3'-untranslated region (UTR) of the SAMD9 gene, miRNA-96 (miR-96) demonstrated significant target-sequence-specific inhibition of the SAMD9 3'-UTR luciferase reporter activity in NSCLC cells. In addition, while NSCLC tumor samples exhibited significantly higher expression levels of miR-96 compared with adjacent normal tissues, the expression levels of SAMD9 were significantly lower than those in adjacent normal tissues. miR-96 and SAMD9 were overexpressed and knocked down in the human NSCLC $\mathrm{H} 358$ and H23 cell lines and the half maximal inhibitory concentration $\left(\mathrm{IC}_{50}\right)$ of cisplatin and cell apoptosis rate under cisplatin treatment were used as measures of cisplatin chemoresistance. The present results identified that overexpression of miR-96 in NSCLC cells markedly decreased SAMD9 expression and cisplatin-induced apoptosis, and increased the cisplatin $\mathrm{IC}_{50}$, which could be eliminated by overexpression of SAMD9. By contrast, knocking down miR-96 in NSCLC cells using antagomir-96 significantly increased SAMD9 expression
\end{abstract}

Correspondence to: $\mathrm{Dr} \mathrm{Lin} \mathrm{Wu}$, Department of Thoracic Medicine, The Affiliated Cancer Hospital of Xiangya School of Medicine, Central South University, 283 Tongzipo Road, Changsha, Hunan 410003, P.R. China

E-mail: wulin-calf@yeah.net

Key words: miR-96, SAMD9, non-small cell lung cancer, cisplatin, chemoresistance, apoptosis and the cisplatin-induced apoptosis and decreased cisplatin $\mathrm{IC}_{50}$, which could be completely reversed by a knockdown of SAMD9. In conclusion, the current study demonstrates that miR-96 targets and downregulates SAMD9 in NSCLC, which decreases cisplatin-induced apoptosis and induces cisplatin chemoresistance in NSCLC cells. The findings of the present study add novel insights into the function of miR-96 and SAMD9 in cancer, as well as into the molecular mechanisms underlying NSCLC chemoresistance.

\section{Introduction}

Non-small cell lung cancer (NSCLC) is the leading cause of mortality due to cancer in the world (1). In locally advanced cancers, chemotherapy and radiation therapy are always incorporated into the treatment regimens of patients (1). Platinum-based adjuvant chemotherapy is a standard treatment for completely resected higher-stage NSCLC (2). Cisplatin is one of the most potent platinum-based chemotherapeutic agents currently in use and is effective as a single agent or in combination with other drugs for the treatment of NSCLC. Cisplatin-based chemotherapy significantly improves the prognosis of patients with NSCLC (3). However, a concerning clinical challenge for cisplatin-based NSCLC chemotherapy is the intrinsic and acquired chemoresistance to the drug (3). Therefore, the identification of factors that contribute to cisplatin chemoresistance in NSCLC may be pivotal for the development of novel therapeutic strategies for this disease.

Deletion of sterile $\alpha$ motif domain-containing 9 (SAMD9) is commonly observed in cells from patients with myeloid leukemia and myelodysplastic syndrome, suggesting that SAMD9 is an inhibitor of tumor progression (4). Ubiquitously expressed in human adult and fetal tissues, SAMD9 is expressed at lower levels in tumors and has been reported to be a potent tumor suppressor gene (5). Overexpression of SAMD9 causes apoptosis and reduced proliferation of malignant cells, whereas downregulation of SAMD9 is associated with increased cellular proliferation and tumor growth in in vivo and in vitro models (5). A recent study has revealed that SAMD9 suppresses tumorigenesis and progression of NSCLC (6).

MicroRNAs (miRNAs) have previously been implicated in oncogenic cell processes, including chemoresistance (7). Lung cancer development is closely correlated with miRNA 
expression (8). Since miRNAs are small non-coding RNA molecules, they post-transcriptionally regulate target gene expression by incomplete base pairing with target mRNAs (9). miRNAs operate through RNA-induced silencing complexes, targeting these complexes to mRNAs where direct destructive cleavage or repression of translation takes place (10). A previous study has demonstrated marked alterations in the miRNA profile in NSCLC compared to adjacent normal tissues (1).

The present study explored the role of miRNA/SAMD9 signaling in regulating cisplatin chemoresistance in NSCLC; to the best of our knowledge this is the first study to do so.

\section{Materials and methods}

Cells lines, plasmid constructs and reagents. The human NSCLC cell lines H358 (catalog no., CRL-5807) and H23 (catalog no., CRL-5800) were purchased from the American Type Culture Collection (ATCC; Manassas, VA, USA). Human SAMD9 3'-untranslated region (UTR) luciferase reporter (catalog no., HmiT013716) and the LucPair Duo Luciferase Assay kit (catalog no., LPFR-M010) were purchased from GeneCopoeia, Inc. (Rockville, MD, USA). Human SAMD9 cDNA clone (catalog no., SC304503) was purchased from OriGene Technologies China (Beijing, China) and subcloned into the pcDNA 3.1 expression vector (catalog no., V790-20; Thermo Fisher Scientific, Inc., Waltham, MA, USA) to generate a pCDNA3.1-SAMD9 expression vector. The 3'-UTR of human SAMD9 was subcloned from the human SAMD9 3'-UTR luciferase reporter and inserted downstream of human SAMD9 cDNA in the pcDNA3.1-SAMD9 expression vector to generate a pcDNA3.1-(SAMD9 cDNA plus 3'-UTR) expression vector. Human SAMD9 short hairpin (sh) RNA lentiviral particles (catalog no., sc-89746-V), control shRNA lentiviral particles-A (catalog no., sc-108080) and goat anti-human polyclonal GAPDH antibody (clone V-18; catalog no., sc-20357; 1:1,000) were purchased from Santa Cruz Biotechnology, Inc. (Dallas, TX, USA). Rabbit anti-human polyclonal anti-SAMD9 antibody (catalog no., HPA021319; $100 \mu \mathrm{l}$ ), puromycin, G418, and cisplatin were purchased from Sigma-Aldrich (St. Louis, MO, USA). Scrambled miR, miR mimics and antagomirs were purchased from NeuroBiotech (Shanghai, China). Lipofectamine ${ }^{\circledR} 2000$ transfection reagent and TRIzol reagent were purchased from Thermo Fisher Scientific, Inc. The TiterTACS in situ apoptosis detection kit (catalog no., 4822-96-K) was purchased from R\&D Systems, Inc. (Minneapolis, MN, USA). The MTT assay kit (catalog no., 30-1010K) was purchased from ATCC. miRNAs that were potentially able to suppress SAMD9 gene (gene ID, NM_017654) expression were selected using TargetScan prediction software (available from http://www.targetscan.org) (10).

Tissue samples. Human NSCLC tumor and adjacent normal lung tissues were obtained from the Tumor Tissue Bank of the Affiliated Cancer Hospital of Xiangya School of Medicine, Central South University (Changsha, Hunan, China). The tissues had been collected from 5 consecutive patients treated at the Affiliated Cancer Hospital of Xiangya School of Medicine, Central South University in July 2005. No patients received chemotherapy or radiotherapy prior to surgery. All NSCLC and adjacent normal tissues (taken $>5 \mathrm{~cm}$ from tumor) were pathologically validated by a pathologist.

Luciferase assay. $\mathrm{H} 358$ and $\mathrm{H} 23$ cells were transfected with luciferase reporter constructs using Lipofectamine 2000 transfection reagent. Luciferase activity was measured $30 \mathrm{~h}$ subsequent to transfection using the LucPair Duo Luciferase Assay kit following the manufacturer's protocol. Each experiment was repeated three times in duplicate.

Reverse transcription-quantitative polymerase chain reaction $(R T-q P C R)$. Total RNA was prepared from the cells using TRIzol reagent. cDNA was synthesized using SuperScript II reverse transcriptase (Thermo Fisher Scientific, Inc.). qPCR was performed using the Applied Biosystems SYBR Green PCR master mix in an Applied Biosystems 7300 real-time PCR System (Thermo Fisher Scientific, Inc.). Applied Biosystems TaqMan microRNA assays (Thermo Fisher Scientific, Inc.), which include RT primers and TaqMan probes, were used to quantify the expression of miR-96. For the measurement of SAMD9 mRNA, the following primers were used: Human SAMD9 forward, 5'-GTGGCCTTTTGTGAT CTCCT-3' and reverse, 5'-CTTATGACTTTCTAACCA CTGA-3'; and human GAPDH forward, 5'-GACTCATGACCA CAGTCCATGC-3' and reverse, 5'-AGAGGCAGGGATGAT GTTCTG-3'. The quantification cycle $\left(\mathrm{C}_{\mathrm{q}}\right)$ for each $\mathrm{PCR}$ product was calculated with the instrument's software and $\mathrm{C}_{\mathrm{q}}$ values were normalized by subtracting the $\mathrm{C}_{\mathrm{q}}$ values of GAPDH. The resulting $\Delta \mathrm{C}_{\mathrm{q}}$ values were used to calculate the relative change in mRNA expression as a ratio, according to the $2^{-\Delta \Delta \mathrm{Cq}}$ method. Each experiment was repeated three times in duplicate.

Western blot analysis. Tissue homogenate or cultured cells were lysed with a hypotonic buffer containing $2 \%$ Nonidet-P and a protease inhibitor cocktail (Sigma-Aldrich) by sonication, which was performed three times for $3 \mathrm{sec}$ on ice. The supernatant obtained subsequent to centrifugation at 2,000 x g for $15 \mathrm{~min}$ at $4^{\circ} \mathrm{C}$, was used for protein concentration determination by the Coomassie blue method and for subsequent steps. Equal amounts of protein were used for each sample and were separated by $8-15 \%$ SDS-polyacrylamide gel and blotted onto a polyvinylidene difluoride microporous membrane (Merck Millipore, Hong Kong, China). The membranes were incubated for $1 \mathrm{~h}$ with a 1:1,000 dilution of primary antibody, and then washed and revealed using bovine anti-goat (catalog no., sc-2350; Santa Cruz Biotechnology, Inc.) or anti-rabbit (catalog no., sc-2370; Santa Cruz Biotechnology, Inc.) secondary antibodies with horseradish peroxidase conjugate (dilution, 1:5,000; $1 \mathrm{~h}$ ). The peroxidase was revealed with an Amersham ECL Western Blotting Detection kit (GE Healthcare Life Sciences, Shanghai, China). Three independent experiments were performed for each western blot analysis.

Transfection and lentiviral transduction. Plasmids, miR mimics and antagomirs were transfected into cells using Lipofectamine 2000 transfection reagent, following the manufacturer's protocol. For stable transfections, pools of stable transfectants of pcDNA3.1-(SAMD9 cDNA plus 3'-UTR) were generated via selection with $700 \mu \mathrm{g} / \mathrm{ml} \mathrm{G} 418$, according 
to the manufacturer's protocol. Lentiviral transduction of SAMD9-shRNA was performed and pools of stable transductants were generated via selection with $4.5 \mu \mathrm{g} / \mathrm{ml}$ puromycin.

Cisplatin chemoresistance assay. The cells were plated in duplicate in 96 -well plates at a density of $5 \times 10^{3}$ cells per well. Subsequent to 24-h incubation, Dulbecco's modified Eagle's medium (Thermo Fisher Scientific, Inc.) was replaced with fresh medium, with or without various concentrations of cisplatin $(0.1,0.25,0.5,1.0,1.5,3.0,6.0,15.0,30.0$ and $55.0 \mathrm{mM})$. Cell viability was assayed $96 \mathrm{~h}$ later using a MTT assay kit following the manufacturer's protocols. The half maximal inhibitory concentration $\left(\mathrm{IC}_{50}\right)$ was defined as the concentration resulting in a $50 \%$ reduction in growth of cells compared to control cells.

Cell apoptosis assay. The cells were cultured at $8 \times 10^{4}$ cells per well in 96-well tissue culture plates and incubated at $37^{\circ} \mathrm{C}$ for 24 or $48 \mathrm{~h}$ with cisplatin $(1 \mu \mathrm{M})$. Cell apoptosis was measured at 24 and $48 \mathrm{~h}$ with a microplate reader-based TiterTACS in situ apoptosis detection kit, according to the manufacturer's protocol (11). The cell apoptosis rate at $24 \mathrm{~h}$ and $48 \mathrm{~h}$ was identified as the percentage of apoptotic cells relative to $100 \%$ cell apoptosis induced by nuclease treatment. Each experiment was repeated for three times in duplicate.

Statistical analysis. Statistical analyses were performed using SPSS for Windows 10.0 (SPSS, Inc., Chicago, IL, USA). All continuous variable values were expressed as the mean \pm standard deviation. Comparison between the means of two groups was performed using Student's $t$-test. Comparison between the means of multiple groups was performed using one-way analysis of variance followed by post hoc pairwise comparisons using Tukey's test. A two-tailed $\mathrm{P}<0.05$ was considered statistically significant.

\section{Results}

miR-96 targets SAMD9 in NSCLC cells. TargetScan prediction software analyzed the 3'-UTR of human SAMD9 gene and cross-referenced the results with a previous study that identified miRNAs that are differentially expressed between NSCLC and adjacent normal lung tissues (1). The previous study revealed that 4 miRNAs were upregulated in NSCLC, consisting of miR-96, miR-374b, miR-182 and miR-1229 (1), and the TargetScan analysis in the present study demonstrated that these 4 miRNAs could potentially target SAMD9. The 4 candidate miRNAs were co-transfected with a SAMD9 3'-UTR luciferase reporter into H358 human NSCLC cells. In total, 7 other miRNAs that demonstrated a highly favorable context+ score ( $\leq-0.4)(12)$ on TargetScan and were not upregulated in NSCLC tumors (1), consisting of miR-298, miR-432, miR-483-3p, miR-626, miR-4628, miR-133b and miR-133a, were also included in the luciferase assays (Fig. 1A). The luciferase activity was measured and normalized to that in cells co-transfected with scramble miR (miR-SCR) and the SAMD9 3'-UTR luciferase reporter $30 \mathrm{~h}$ subsequent to transfection. As demonstrated by Fig. 1A, among the miRNAs tested, only miR-96 significantly decreased the luciferase activity (cut off value, 1.0), suggesting that miR-96 targeted SAMD9.
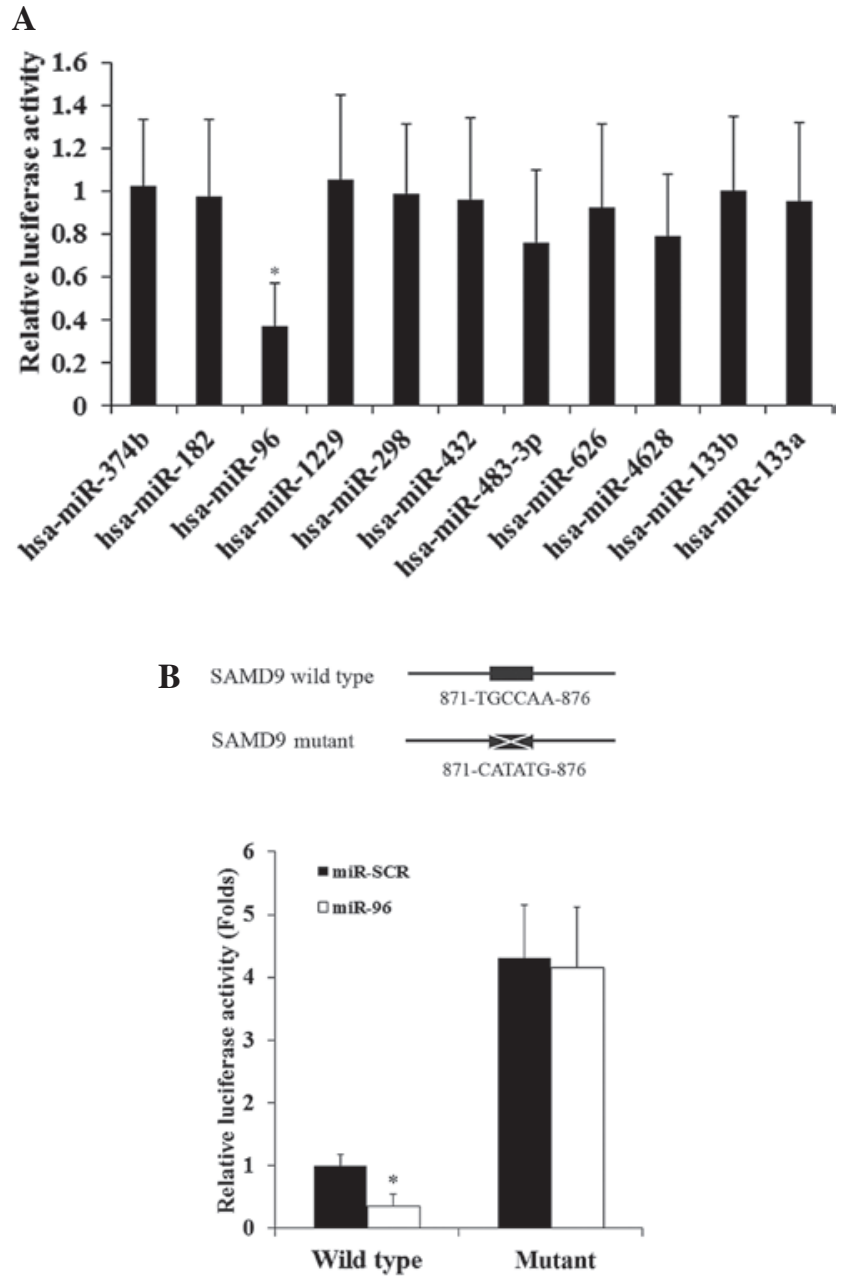

Figure 1. Effect of selected miRNAs on the SAMD9 3'-UTR luciferase reporter in NSCLC cells. (A) In total, 11 miRNAs potentially able to regulate SAMD9 were selected based on TargetScan prediction software. The miRNA mimics were co-transfected with a SAMD9 3'-UTR luciferase reporter in human NSCLC H358 cells. In total, $30 \mathrm{~h}$ subsequent to transfection, the luciferase activity was measured. ${ }^{*} \mathrm{P}<0.05$ vs. 1.0 (cut off value). (B) Luciferase activities were measured in $\mathrm{H} 358$ cells co-transfected with miR-96 mimics and the SAMD9 3'-UTR luciferase reporter with wild-type or mutant miR-96-binding sequence. Cells co-transfected with miR-SCR instead of miR-96 mimics was used as a negative control. The luciferase activity was expressed as fold changes compared to cells co-transfected with miR-SCR and the wild-type SAMD9 3'-UTR luciferase reporter (designated as 1$) .{ }^{*} \mathrm{P}<0.05$ vs. miR-SCR. UTR, untranslated region; NSCLC, non-small cell lung cancer; miR, microRNA; SAMD9, sterile $\alpha$ motif domain-containing 9; miR-SCR, scramble miR.

To demonstrate a direct interaction between miR-96 and SAMD9, the potential binding sequence for miR-96 in the 3'-UTR of the SAMD9 gene, as predicted by TargetScan, was mutated to generate a mutant SAMD9 3'-UTR luciferase reporter (Fig. 1B). H358 cells were co-transfected with miR-96 or miR-SCR and the wild-type or mutant SAMD9 3'-UTR luciferase reporter. Fig. 1B reveals that miR-96 decreased the luciferase activity of the wild-type SAMD9 3'-UTR luciferase reporter by $\sim 65 \%$ compared with miR-SCR. However, there was no significant difference between the effects of miR-96 and miR-SCR on the mutant SAMD9 3'-UTR luciferase reporter. The results suggest that miR-96 may directly target SAMD9 in NSCLC cells. In addition, the mutant SAMD9 3'-UTR luciferase reporter showed markedly higher luciferase activity compared to the wild-type, 
A

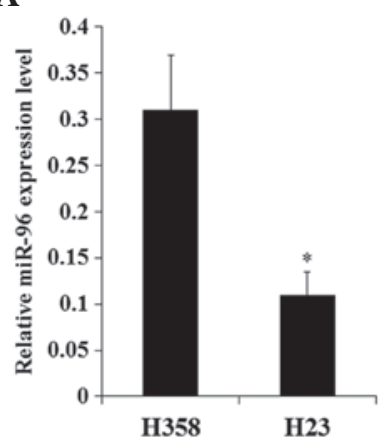

B

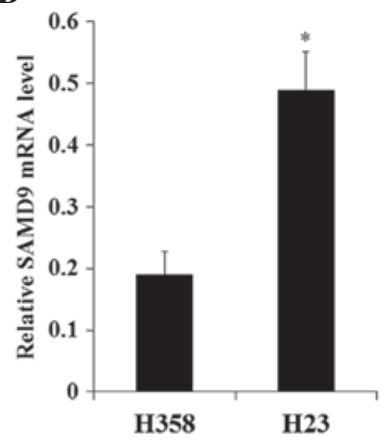

C
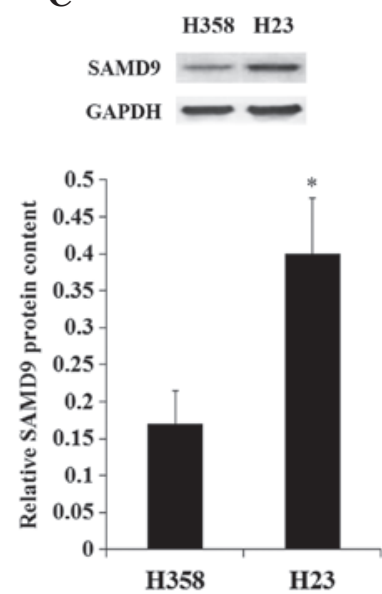

Figure 2. Expression of miR-96 and SAMD9 in NSCLC cells. In H358 and H23 NSCLC cells, the expression levels of (A) miR-96 and (B) SAMD9 mRNA were determined with reverse transcription-quantitative polymerase chain reaction. (C) SAMD9 protein levels were determined by western blot analysis. Density of the SAMD9 blots was normalized against that of GAPDH to obtain a relative blot density to indicate the relative SAMD9 protein content. "P<0.05 vs. H358. NSCLC, non-small cell lung cancer; miR, microRNA; SAMD9, sterile $\alpha$ motif domain-containing 9.

possibly due to mutation of the miR-96 binding sequence eliminating the effect of constitutively expressed miR-96 on the luciferase reporter.

miR-96 inhibits the expression of SAMD9 in NSCLC cells. The constitutive expression of miR-96 and SAMD9 in NSCLC cell lines was investigated. The constitutive expression level of miR-96 in H358 cells was increased $~ 3$-fold compared with H23 cells (Fig. 2A). By contrast, the expression of SAMD9 at the mRNA and protein levels in $\mathrm{H} 358$ cells was $<50 \%$ that in H23 cells (Fig. 2B). The results suggest a negative association between miR-96 and SAMD9 in NSCLC cells. In addition, the expression levels of miR-96 and SAMD9 were determined in NSCLC and adjacent normal lung tissues in 5 consecutive patients, who received no chemotherapy or radiotherapy prior to surgery. As demonstrated in Fig. 3, while NSCLC tumor samples exhibited significantly increased expression levels of miR-96 compared with adjacent normal tissues, the expression levels of SAMD9 were significantly decreased compared to those in adjacent normal tissues. The in vitro and in vivo results suggest that miR-96 is negatively associated with SAMD9 in NSCLC.

To determine the regulatory effects of miR-96 on SAMD9 expression in NSCLC cells, miR-96 was overexpressed

A
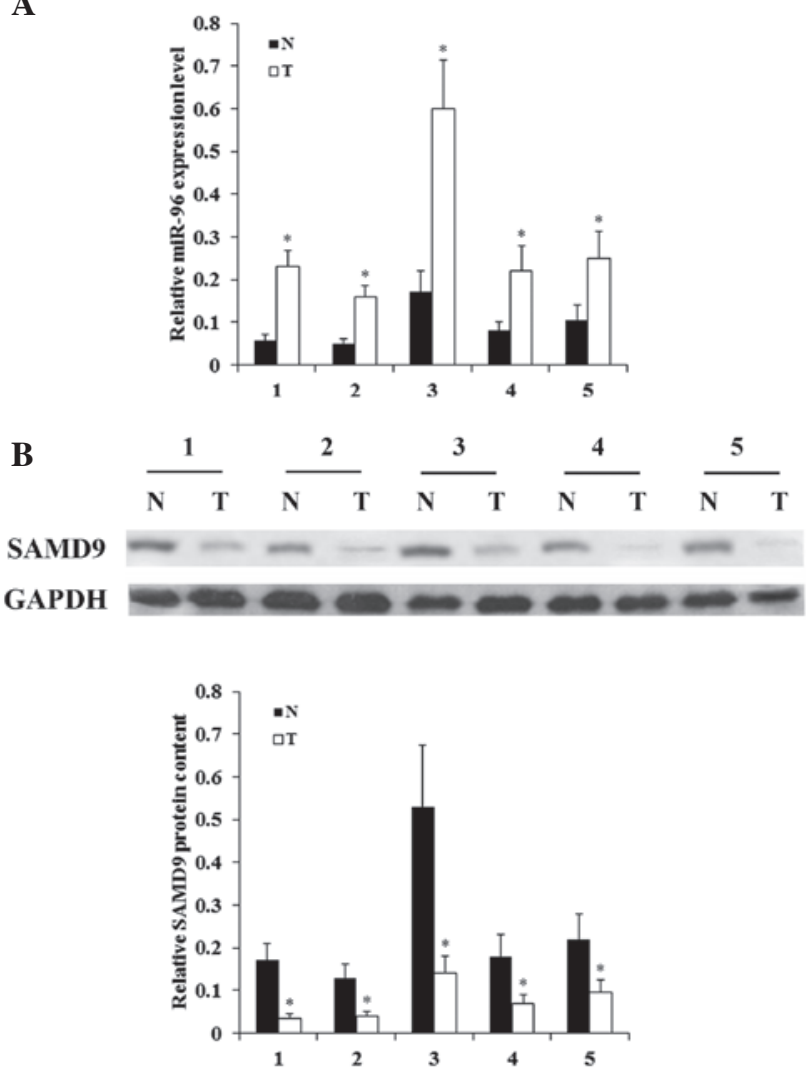

Figure 3. Expression of miR-96 and SAMD9 in NSCLC and adjacent normal lung tissues. The expression levels of (A) miR-96 and (B) SAMD9 protein in $\mathrm{T}$ and $\mathrm{N}$ lung tissues from 5 consecutive patients were determined with reverse transcription-quantitative polymerase chain reaction and western blot analysis, respectively. In western blot analysis, the density of the SAMD9 blots was normalized against that of GAPDH to obtain a relative blot density to indicate the relative SAMD9 protein content. ${ }^{*} \mathrm{P}<0.05$ vs. N. NSCLC, non-small cell lung cancer; miR microRNA; SAMD9, sterile $\alpha$ motif domain-containing 9; T, NSCLC tumor; N, adjacent normal tissue.

and knocked down in $\mathrm{H} 358$ and $\mathrm{H} 23$ cells. Overexpression of miR-96 decreased the constitutive mRNA level of SAMD9 by $\sim 60$ and 55\% in H358 and H23 cells, respectively (Fig. 4). By contrast,knocking down miR-96 with antagomir-96increased the constitutive mRNA level of SAMD9by 3-and2-fold in H358 and H23 cells, respectively (Fig. 4). Compared with the controls, stable overexpression of SAMD9 (SAMD9 cDNA + 3'-UTR) in $\mathrm{H} 358$ cells increased the mRNA level of SAMD9 by $>4.5$ fold, which was reversed by overexpression of miR-96 (Fig. 4A). Stable transduction of $\mathrm{H} 23$ cells with lentiviral SAMD9-shRNA knocked down the SAMD9 mRNA level by $\sim 80 \%$, which was only partially reversed by antagomir-96 (Fig. 4B). Similar data trends were observed at the protein level of SAMD9 in H358 and H23 cells (Fig. 5).

Effect of miR-96/SAMD9 signaling on cisplatin chemoresistance in NSCLC cells. To explore the individual effect and interaction between miR-96 and SAMD9 on NSCLC chemoresistance, the cisplatin $\mathrm{IC}_{50}$ in NSCLC cells was investigated. An increased $\mathrm{IC}_{50}$ value was considered to correspond with clinical chemoresistance to cisplatin (13). As demonstrated in Fig. 6A, subsequent to $96 \mathrm{~h}$ of cisplatin treatment, the cisplatin $\mathrm{IC}_{50}$ for $\mathrm{H} 358$ cells was $1.31 \mu \mathrm{M}$. 
A
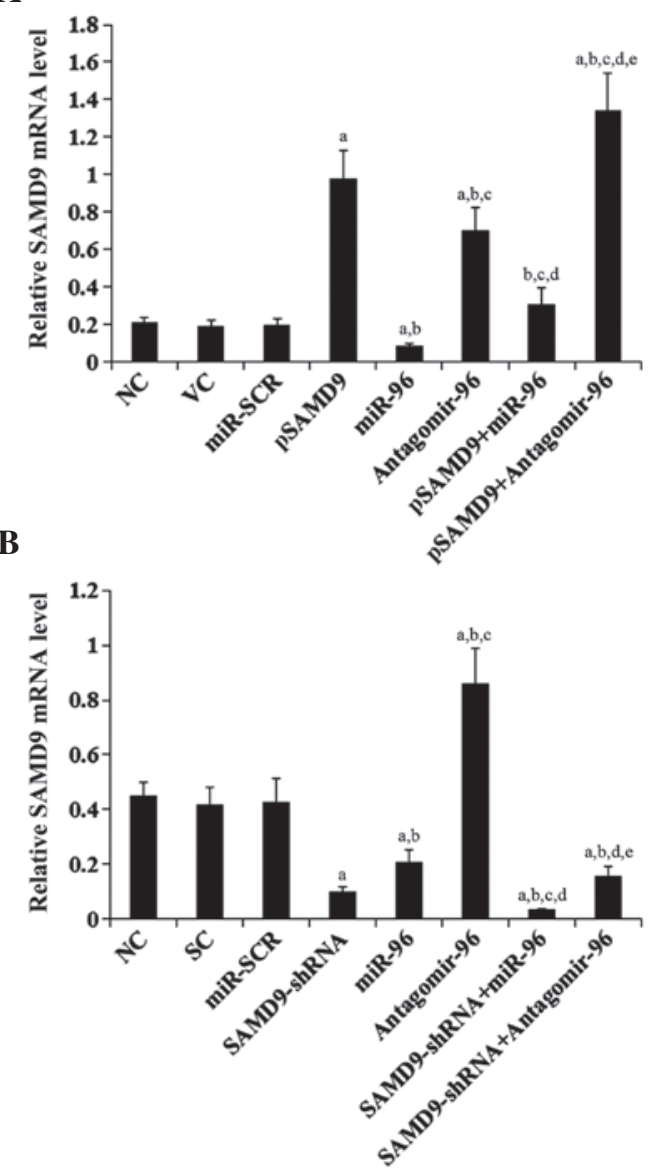

Figure 4. Effect of miR-96 on SAMD9 mRNA levels in non-small cell lung cancer cells (A) In H358 cells, the SAMD9 mRNA level was determined with RT-qPCR in NC, cells stably transfected with VC, transfected with miR-SCR, stably transfected with pSAMD9, transfected with miR-96 mimics, transfected with antagomir-96, stably transfected with pSAMD9 + miR-96, and stably transfected with pSAMD9 + antagomir-96. (B) In H23 cells, the SAMD9 mRNA level was determined with RT-qPCR in NC cells and cells stably transduced with SC, transfected with miR-SCR, stably transduced with SAMD9-shRNA, transfected with miR-96 mimics, transfected with antagomir- 96, stably transduced with SAMD9-shRNA and transiently transfected with miR-96 mimics, and stably transduced with SAMD9-shRNA and transiently transfected with antagomir-96. In (A) $\mathrm{H} 358$ cells, ${ }^{\text {a }}<<0.05$ vs. NC, VC and miR-SCR; ${ }^{b} \mathrm{P}<0.05$ vs. pSAMD9; ${ }^{\circ} \mathrm{P}<0.05$ vs. miR-96; ${ }^{\mathrm{d}} \mathrm{P}<0.05$ vs. antagomir- 96 ; ${ }^{\mathrm{e}} \mathrm{P}<0.05$ vs. pSAMD $9+$ miR-96. In (B) $\mathrm{H} 23$ cells, ${ }^{a} \mathrm{P}<0.05$ vs. NC, $\mathrm{SC}$ and $\mathrm{miR}-\mathrm{SCR}$; ${ }^{\mathrm{b}} \mathrm{P}<0.05$ vs. SAMD9-shRNA; ${ }^{\mathrm{c}} \mathrm{P}<0.05$ vs. $\mathrm{miR}-96$; ${ }^{\mathrm{d}} \mathrm{P}<0.05$ vs. antagomir- 96 ; ${ }^{\mathrm{e}} \mathrm{P}<0.05$ vs. SAMD9-shRNA + miR-96. miR, microRNA; SAMD9, sterile $\alpha$ motif domain-containing 9; RT-qPCR, reverse transcription-quantitative polymerase chain reaction; NC, normal control cells; VC, pcDNA3.1 plasmid; miR-SCR, scramble miR; UTR, untranslated region; pSAMD9, pcDNA3.1-(SAMD9 cDNA plus UTR) plasmid; pSAMD9 + miR-96, pcDNA3.1-(SAMD9 cDNA plus UTR) plasmid and transiently transfected with miR-96 mimics; pSAMD9 + antagomir-96, pcDNA3.1-(SAMD9 cDNA plus UTR) plasmid and transiently transfected with antagomir-96; SC, scramble control short hairpin RNA.

Overexpression of miR-96 increased the $\mathrm{IC}_{50}$ to $3.78 \mu \mathrm{M}$, which was eliminated by overexpression of SAMD9 (Fig. 6A). By contrast, antagomir-96 decreased the $\mathrm{IC}_{50}$ to $0.42 \mu \mathrm{M}$, which was enhanced by overexpression of SAMD9 (Fig. 6A). In $\mathrm{H} 23$ cells, the $\mathrm{IC}_{50}$ for cisplatin was $0.74 \mu \mathrm{M}$ (Fig. 6B). Overexpression of miR-96 increased the $\mathrm{IC}_{50}$ to $1.52 \mu \mathrm{M}$, which was enhanced by knockdown of SAMD9 (Fig. 6B). By contrast, antagomir-96 decreased the $\mathrm{IC}_{50}$ to $0.43 \mu \mathrm{M}$, which was eliminated by knockdown of SAMD9 (Fig. 6B).
A

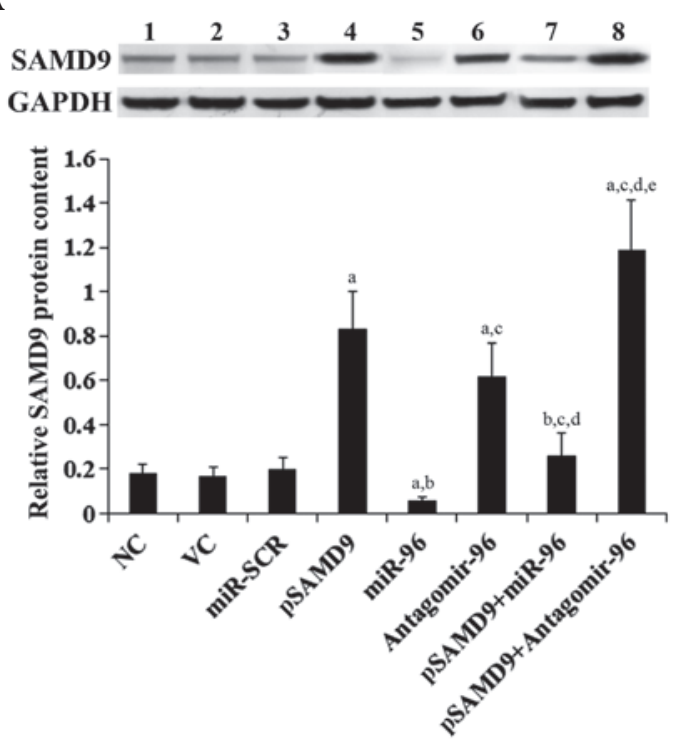

B
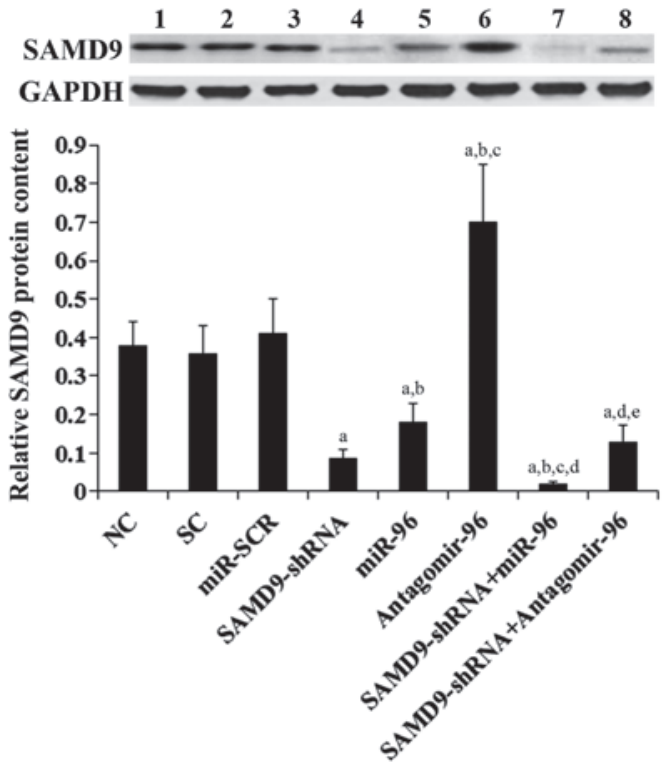

Figure 5. Effect of miR-96 on SAMD9 protein levels in non-small cell lung cancer cells. (A) In H358 cells, the SAMD9 protein level was determined with western blot analysis in NC (lane 1), cells stably transfected with VC (lane 2), transfected with miR-SCR (lane 3), stably transfected with pSAMD9 (lane 4), transfected with miR-96 mimics (lane 5), transfected with antagomir-96 (lane 6), stably transfected with pSAMD9 + miR-96 (lane 7), and stably transfected with pSAMD9 + antagomir-96 (lane 8). (B) In $\mathrm{H} 23$ cells, the SAMD9 protein level was determined with western blot analysis in NC cells (lane 1), cells stably transduced with SC (lane 2), transfected with miR-SCR (lane 3), stably transduced with SAMD9-shRNA (lane 4), transfected with miR-96 mimics (lane 5), transfected with antagomir-96 (lane 6), stably transduced with SAMD9-shRNA and transiently transfected with miR-96 mimics (lane 7), and stably transduced with SAMD9-shRNA and transiently transfected with antagomir-96 (lane 8). Density of the SAMD9 blot was normalized against that of GAPDH to obtain a relative blot density to represent relative SAMD9 protein content. In (A) H358 cells, ${ }^{\mathrm{a}} \mathrm{P}<0.05$ vs. NC, VC and miR-SCR; ${ }^{\mathrm{b}} \mathrm{P}<0.05$ vs. pSAMD9; ${ }^{\mathrm{C}} \mathrm{P}<0.05$ vs. miR-96; ${ }^{\mathrm{d}} \mathrm{P}<0.05$ vs. antagomir-96; ${ }^{\mathrm{e}} \mathrm{P}<0.05$ vs. pSAMD9 + miR-96. In (B) H23 cells, ${ }^{\mathrm{a}} \mathrm{P}<0.05$ vs. NC, SC and miR-SCR; ${ }^{\mathrm{b}} \mathrm{P}<0.05$ vs. SAMD9-shRNA; ${ }^{\mathrm{c}} \mathrm{P}<0.05$ vs. $\mathrm{miR}-96$; ${ }^{\mathrm{d}} \mathrm{P}<0.05$ vs. antagomir-96; ${ }^{\mathrm{e}} \mathrm{P}<0.05$ vs. SAMD9-shRNA + miR-96. miR, microRNA; SAMD9, sterile $\alpha$ motif domain-containing 9; NC, normal control cells; VC, pcDNA3.1 plasmid; miR-SCR, scramble miR; UTR, untranslated region; pSAMD9, pcDNA3.1-(SAMD9 cDNA plus UTR) plasmid; pSAMD9 + miR-96, pcDNA3.1-(SAMD9 cDNA plus UTR) plasmid and transiently transfected with miR-96 mimics; pSAMD9 + antagomir-96, pcDNA3.1-(SAMD9 cDNA plus UTR) plasmid and transiently transfected with antagomir-96; SC, scramble control short hairpin RNA. 
A
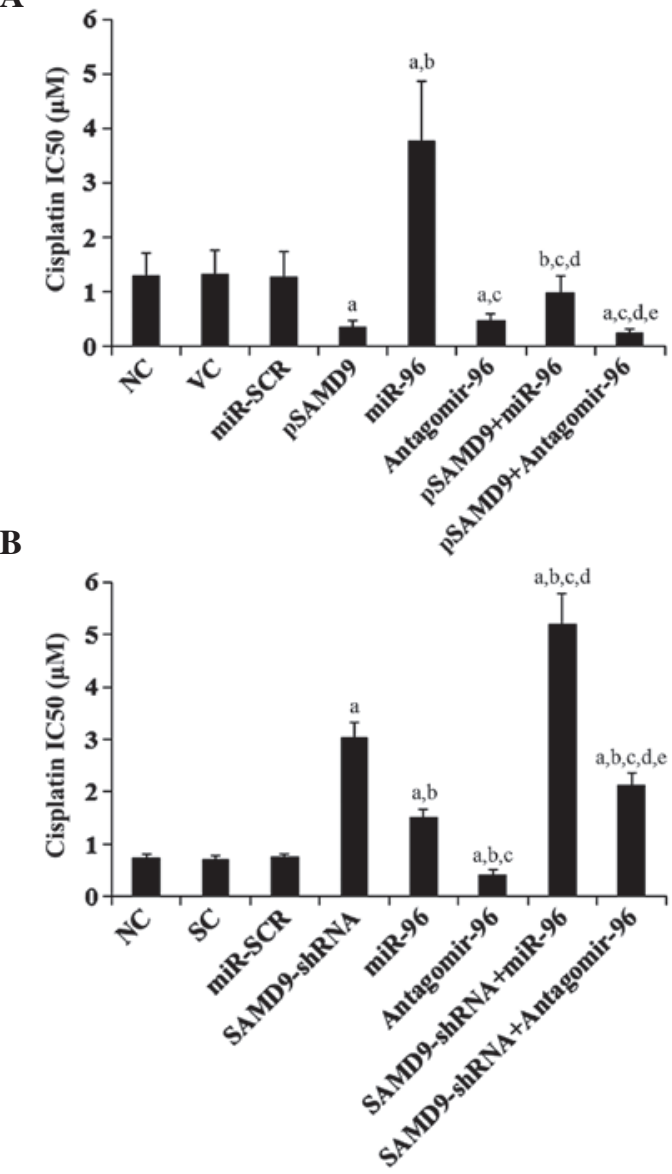

Figure 6. Effect of miR-96/SAMD9 signaling on cisplatin chemoresistance in non-small cell lung cancer cells. $\mathrm{H} 358$ and $\mathrm{H} 23$ cells were treated with or without various concentrations of cisplatin $(0.1,0.25,0.5,1.0,1.5,3.0,6.0$ $15.0,30.0$ and $55.0 \mathrm{mM}$ ) for $96 \mathrm{~h}$. (A) $\mathrm{In} \mathrm{H} 358$ cells, $\mathrm{IC}_{50}$ was determined in NC, cells stably transfected with VC, transfected with miR-SCR, stably transfected with pSAMD9, transfected with miR-96 mimics, transfected with antagomir-96, stably transfected with pSAMD9 + miR-96, and stably transfected with pSAMD9 + antagomir-96. (B) In $\mathrm{H} 23$ cells, $\mathrm{IC}_{50}$ was determined in NC, cells stably transduced with SC, transfected with miR-SCR, stably transduced with SAMD9-shRNA, transfected with miR-96 mimics, transfected with antagomir-96, stably transduced with SAMD9-shRNA and transiently transfected with miR-96 mimics, and stably transduced with SAMD9-shRNA and transiently transfected with antagomir-96. In (A) H358 cells, ${ }^{a} \mathrm{P}<0.05$ vs. NC, VC and miR-SCR; ${ }^{\mathrm{P}} \mathrm{P}<0.05$ vs. pSAMD9; ${ }^{\mathrm{C}} \mathrm{P}<0.05$ vs. miR-96; ${ }^{\mathrm{d}} \mathrm{P}<0.05$ vs. antagomir- 96 ; ${ }^{\mathrm{e}} \mathrm{P}<0.05$ vs. pSAMD9 + miR-96. In (B) H23 cells, ${ }^{\mathrm{a}} \mathrm{P}<0.05$ vs. NC, $\mathrm{SC}$ and miR-SCR; ${ }^{\mathrm{b}} \mathrm{P}<0.05$ vs. SAMD9-shRNA; ${ }^{\mathrm{c}} \mathrm{P}<0.05$ vs. miR- 96 ; ${ }^{\mathrm{d}} \mathrm{P}<0.05$ vs. antagomir- 96 ; ${ }^{\mathrm{e}} \mathrm{P}<0.05$ vs. SAMD9-shRNA + miR-96. miR, microRNA; SAMD9, sterile $\alpha$ motif domain-containing 9; $\mathrm{IC}_{50}$, the half maximal inhibitory concentration; NC, normal control cells; VC, pcDNA3.1 plasmid; miR-SCR, scramble miR; UTR, untranslated region; pSAMD9, pcDNA3.1-(SAMD9 cDNA plus UTR) plasmid; pSAMD9 + miR-96, pcDNA3.1-(SAMD9 cDNA plus UTR) plasmid and transiently transfected with miR-96 mimics; pSAMD9 + antagomir-96, pcDNA3.1-(SAMD9 cDNA plus UTR) plasmid and transiently transfected with antagomir-96; SC, scramble control short hairpin RNA.

In addition, overexpression of SAMD9 decreased the $\mathrm{IC}_{50}$ for cisplatin to $0.35 \mu \mathrm{M}$ in $\mathrm{H} 358$ cells (Fig. 6A), and knockdown of SAMD9 increased the $\mathrm{IC}_{50}$ to $3.05 \mu \mathrm{M}$ in $\mathrm{H} 23$ cells (Fig. 6B).

Effect of miR-96/SAMD9 signaling on cisplatin-induced apoptosis in NSCLC cells. The individual effect and interaction between miR-96 and SAMD9 on cisplatin-induced apoptosis in NSCLC cells was investigated. As revealed in Fig. 7, in
A

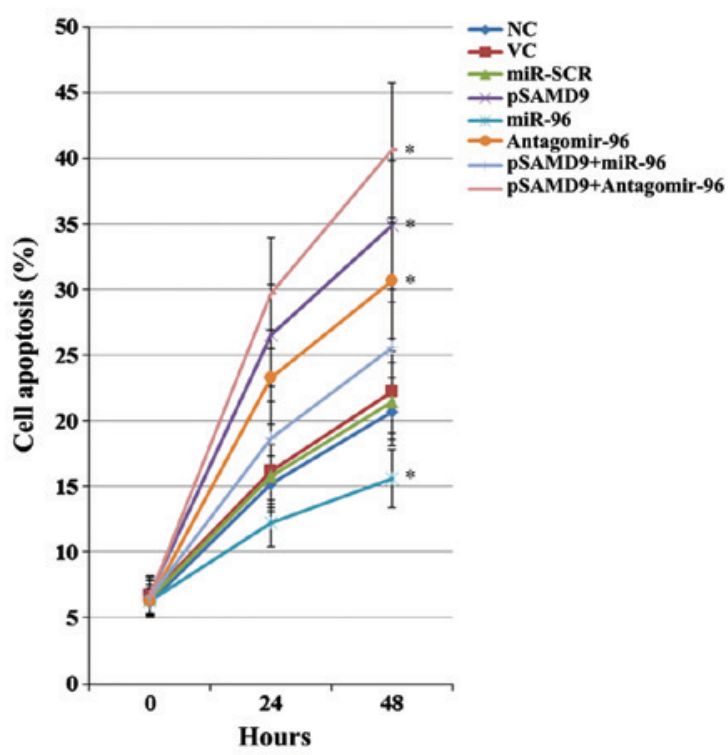

B

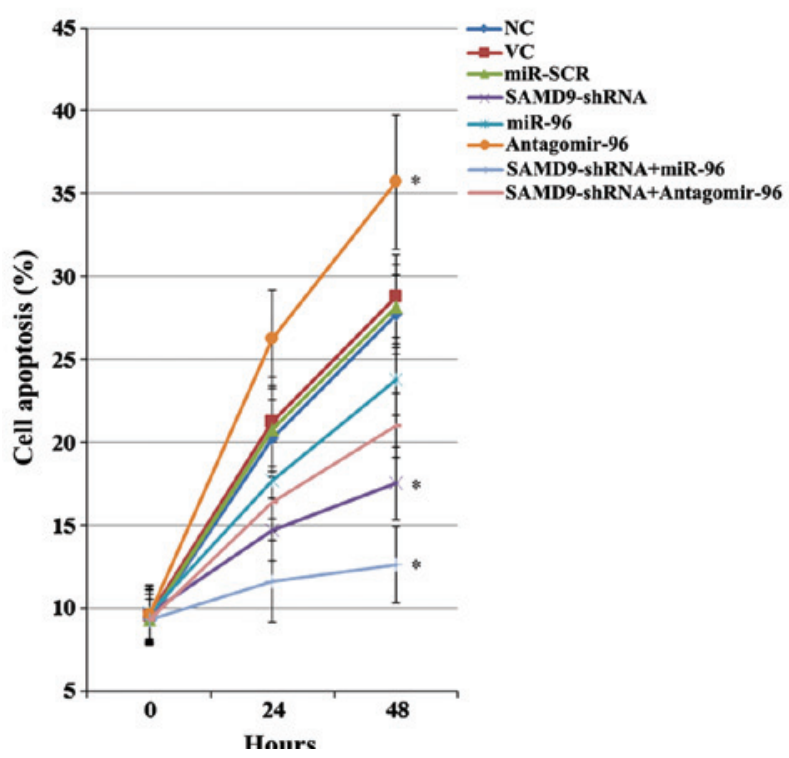

Figure 7. Effect of miR-96/SAMD9 signaling on cisplatin-induced apoptosis in non-small cell lung cancer (NSCLC) cells. H358 and H23 cells were treated with cisplatin $(1 \mu \mathrm{M})$ for $24 \mathrm{~h}$ and $48 \mathrm{~h}$. Apoptosis was measured with a microplate reader-based TiterTACS in situ apoptosis detection kit. (A) In H358 cells, apoptosis was determined in NC, cells stably transfected with VC, transfected with miR-SCR, stably transfected with pSAMD9, transfected with miR-96 mimics, transfected with antagomir-96, stably transfected with pSAMD9 + miR-96, and stably transfected with pSAMD9 + antagomir-96. (B) In H23 cells, apoptosis was determined in $\mathrm{NC}$, cells stably transduced with SC, transfected with miR-SCR, stably transduced with SAMD9-shRNA, transfected with miR-96 mimics, transfected with antagomir-96, stably transduced with SAMD9-shRNA and transiently transfected with miR-96 mimics, and stably transduced with SAMD9-shRNA and transiently transfected with antagomir-96. The cell apoptosis rates at $24 \mathrm{~h}$ and $48 \mathrm{~h}$ were shown as the percentage of apoptotic cells (as compared to $100 \%$ cell apoptosis induced by nuclease treatment). In (A) H358 cells, ${ }^{*} \mathrm{P}<0.05$ vs. NC, VC and miR-SCR. In (B) H23 cells, "P<0.05 vs. NC, SC and miR-SCR. miR microRNA; SAMD9, sterile $\alpha$ motif domain-containing $9 ; \mathrm{IC}_{50}$, the half maximal inhibitory concentration; $\mathrm{NC}$, normal control cells; VC, pcDNA3.1 plasmid; miR-SCR, scramble miR; UTR, untranslated region; pSAMD9, pcDNA3.1-(SAMD9 cDNA plus UTR) plasmid; pSAMD9 + miR-96, pcDNA3.1-(SAMD9 cDNA plus UTR) plasmid and transiently transfected with miR-96 mimics; pSAMD9 + antagomir-96, pcDNA3.1-(SAMD9 cDNA plus UTR) plasmid and transiently transfected with antagomir-96; SC, scramble control shRNA. 
untreated $\mathrm{H} 358$ and $\mathrm{H} 23$ cells at $0 \mathrm{~h}$, overexpression and knockdown of SAMD9 and/or miR-96 demonstrated no significant effect on NSCLC cell apoptosis. Subsequent to $48 \mathrm{~h}$ of cisplatin $(1 \mu \mathrm{M})$ treatment, the percentage of apoptotic cells in H358 cells increased to $21.5 \%$ (Fig. 7A). Overexpression of miR-96 decreased cell apoptosis to $15.6 \%$, which was eliminated by overexpression of SAMD9 (Fig. 7A). Antagomir-96 increased cell apoptosis to 30.7\%, which was enhanced by overexpression of SAMD9 (Fig. 7A). In $\mathrm{H} 23$ cells, the cell apoptosis rate subsequent to $48 \mathrm{~h}$ of treatment with $1 \mu \mathrm{M}$ cisplatin was $\sim 28 \%$ (Fig. 7B). Overexpression of miR-96 decreased cell apoptosis to $23.8 \%$, which was enhanced by the knockdown of SAMD9 (Fig. 6B). By contrast, antagomir-96 increased the cell apoptosis rate to $35.7 \%$, which was eliminated by knockdown of SAMD9 (Fig. 7B). In addition, overexpression of SAMD9 increased the cell apoptosis rate to $34.9 \%$ in H358 cells (Fig. 7A), and knockdown of SAMD9 decreased the cell apoptosis rate to $17.5 \%$ in $\mathrm{H} 23$ cells (Fig. 7B).

\section{Discussion}

Platinum-based therapy is the mainstay of chemotherapy for NSCLC (3). Although platinum-based adjuvant chemotherapy significantly increases the overall 5-year survival rate of NSCLC patients, the treatment regimen fails in $\sim 50 \%$ of patients, due to intrinsic and acquired cisplatin resistance (2). SAMD9 is reportedly a potent tumor suppressor gene (5) that has been demonstrated to inhibit tumorigenesis and progression of NSCLC (6). The present study reports, to the best of our knowledge, the first evidence that SAMD9 increases cisplatin-induced apoptosis and decreases cisplatin chemoresistance in NSCLC cells.

miRNAs have been identified to play important roles in the regulation of cancer chemoresistance (7). The aim of the current study was to identify miRNAs that regulate SAMD9 expression, and the results revealed that miR-96 directly targets and downregulates SAMD9 in NSCLC. The results are supported by the following in vivo and in vitro findings: A negative association between miR-96 and SAMD9 expression in NSCLC and adjacent normal lung tissues; target-sequence-specific inhibition of the SAMD9 3'-UTR luciferase reporter by miR-96 in NSCLC cells; and alteration of SAMD9 expression by overexpression and inhibition of miR-96 in NSCLC cells.

The cisplatin $\mathrm{IC}_{50}$ was employed as a measure of cisplatin chemoresistance in NSCLC cells. A higher $\mathrm{IC}_{50}$ value was considered to be associated with clinical chemoresistance to cisplatin, which is one of the most potent platinum-based chemotherapeutic agents currently in use (3). miR-96/SAMD9 signaling significantly altered cisplatin chemoresistance in NSCLC cells. In the presence of cisplatin, antagomir-96 significantly enhanced cisplatin-induced apoptosis and decreased cisplatin chemoresistance in NSCLC cells, suggesting that inhibition of miR-96 may be a potential novel strategy to enhance chemotherapy for NSCLC. The effects of antagomir-96 were reversed by knockdown of SAMD9 and enhanced by overexpression of SAMD9, indicating that miR-96 promotes NSCLC cell resistance to cisplatin mainly by downregulating SAMD9, or antagomir-96 suppresses cisplatin chemoresistance by upregulating SAMD9.

miR-96 has been shown to promote proliferation and chemoresistance by downregulating reversion-inducing-cysteine-rich protein with Kazal motifs (RECK) in esophageal cancer (14). A recent study has reported that miR-96 inhibits NSCLC cell apoptosis by targeting forkhead box O3 (FOXO3) (15). In addition, another recent study has suggested that miR-96 acts as a tumor suppressor in pancreatic cancer, and therefore, may act as a useful therapeutic target for the development of novel anticancer therapies (16). In the present study, miR-96 has been revealed to inhibit cisplatin-induced apoptosis and induce cisplatin chemoresistance in NSCLC cells by inhibiting the expression of SAMD9. Overall, the results suggest that miR-96 plays a dual role in cancer malignancy and chemoresistance, depending on tissue specificity. The enhancing effect of miR-96 on NSCLC chemoresistance, through downregulation of SAMD9 expression, is a novel function of this miR and miR-96/SAMD9 signaling may be a novel mechanism involved in the development of NSCLC chemoresistance. How and whether SAMD9, FOXO3 and possibly RECK interact with each other to affect cisplatin chemoresistance in NSCLC cells may be a notable topic for future studies.

Cisplatin elicits DNA repair mechanisms by crosslinking DNA that in turn, activates apoptosis when DNA repair is impossible (17). In the present study, only the effect of miR-96/SAMD9 signaling on cisplatin chemoresistance in NSCLC cells was investigated; therefore, it is unclear whether miR-96/SAMD9 is involved in chemoresistance to other types of chemotherapy agents for NSCLC. Additional studies with various types of chemotherapy agents and NSCLC cell lines may resolve this issue. Furthermore, since SAMD9 has been associated with aggressive fibromatosis and breast and colon cancers (6), it is worth defining the role of miR-96/SAMD9 signaling in other cancers.

In conclusion, the present study has demonstrated that miR-96 targets and downregulates SAMD9 in NSCLC, which decreases cisplatin-induced apoptosis and induces cisplatin chemoresistance in NSCLC cells. The current study provides novel insights into the functions of miR-96 and SAMD9 in cancer and the molecular mechanisms underlying NSCLC chemoresistance.

\section{References}

1. Ma L, Huang Y, Zhu W, et al: An integrated analysis of miRNA and mRNA expressions in non-small cell lung cancers. PLoS One 6: e26502, 2011.

2. Merk J, Rolff J, Dorn C, Leschber G and Fichtner I: Chemoresistance in non-small-cell lung cancer: Can multidrug resistance markers predict the response of xenograft lung cancer models to chemotherapy? Eur J Cardiothorac Surg 40: e29-e33, 2011.

3. Chang A: Chemotherapy, chemoresistance and the changing treatment landscape for NSCLC. Lung Cancer 71: 3-10, 2011.

4. Asou H, Matsui H, Ozaki Y, et al: Identification of a common microdeletion cluster in 7q21.3 subband among patients with myeloid leukemia and myelodysplastic syndrome. Biochem Biophys Res Commun 383: 245-251, 2009.

5. Li CF, MacDonald JR, Wei RY, et al: Human sterile alpha motif domain 9, a novel gene identified as down-regulated in aggressive fibromatosis, is absent in the mouse. BMC Genomics 8: 92, 2007. 
6. Ma Q, Yu T, Ren YY, Gong T and Zhong DS: Overexpression of SAMD9 suppresses tumorigenesis and progression during non small cell lung cancer. Biochem Biophys Res Commun 454 157-161, 2014.

7. Feng B, Wang R and Chen LB: Review of miR-200b and cancer chemosensitivity. Biomed Pharmacother 66: 397-402, 2012.

8. Van Den Broeck A, Ozenne P, Eymin B and Gazzeri S: Lung cancer: A modified epigenome. Cell Adh Migr 4: 107-113, 2010.

9. Bartel DP: MicroRNAs: Genomics, biogenesis, mechanism, and function. Cell 116: 281-297, 2004.

10. Filipowicz W, Jaskiewicz L, Kolb FA and Pillai RS: Post-transcriptional gene silencing by siRNAs and miRNAs. Curr Opin Struct Biol 15: 331-341, 2005.

11. Byun K, Bayarsaikhan E, Kim CY, Mook-Jung I, Paek SH, Kim SU, Yamamoto T, Won MH, Song BJ, et al: Induction of neuronal death by microglial AGE-albumin: Implications for Alzheimer's disease. PLoS ONE 7: e37917, 2012

12. Garcia DM, Baek D, Shin C, Bell GW, Grimson A and Bartel DP: Weak seed-pairing stability and high target-site abundance decrease the proficiency of 1sy-6 and other microRNAs. Nat Struct Mol Biol 18: 1139-1146, 2011.
13. Liu M, Wang J, Huang H, Hou J, Zhang B and Wang A: miR-181a-Twist1 pathway in the chemoresistance of tongue squamous cell carcinoma. Biochem Biophys Res Commun 441: 364-370, 2013.

14. Xia H, Chen S, Chen K, Huang H and Ma H: MiR-96 promotes proliferation and chemo- or radioresistance by down-regulating RECK in esophageal cancer. Biomed Pharmacother 68: 951-958, 2014

15. Li J, Li P, Chen T, Gao G, Chen X, Du Y, Zhang R, Yang R, Zhao W, Dun S, Gao F, et al: Expression of microRNA-96 and its potential functions by targeting FOXO3 in non-small cell lung cancer. Tumour Biol 36: 685-692, 2015.

16. Feng J, Yu J, Pan X, Li Z, Chen Z, Zhang W, Wang B, Yang L, $\mathrm{Xu} \mathrm{H}$, Zhang $\mathrm{G}$ and $\mathrm{Xu} \mathrm{Z}$ : HERG1 functions as an oncogene in pancreatic cancer and is downregulated by miR-96. Oncotarget 5: 5832-5844, 2014

17. Rosenberg B, VanCamp L, Trosko JE and Mansour VH: Platinum compounds: A new class of potent antitumour agents. Nature 222: 385-386, 1969. 